\title{
The inviscid impingement of a jet with arbitrary velocity profile
}

\author{
Denis J. Phares ${ }^{\text {a) }}$ and Gregory T. Smedley \\ Environmental Engineering Science, California Institute of Technology, Pasadena, California 91125 \\ Richard C. Flagan \\ Chemical Engineering, California Institute of Technology, Pasadena, California 91125
}

(Received 24 February 1999; accepted 3 May 2000)

\begin{abstract}
Accurate determination of wall shear stress and heat and mass transfer rates under an impinging jet requires careful analysis of the boundary layer at the impingement surface due to the large pressure gradients near the stagnation point. Modeling the inviscid flow just outside the boundary layer provides the boundary conditions necessary for such an analysis. Previous inviscid models have considered only a small subset of possible jet velocity profiles and with limited spatial resolution. In the present work, analytical solutions to the stream-vorticity equation for two-dimensional and axisymmetric impingement flow with arbitrary velocity profile are found in terms of a surface integral involving the vorticity function, allowing an iterative determination of the stream function throughout the impingement region. Surface pressure distributions and streamline plots are calculated for various impinging jet configurations, including plane, round, and annular jet nozzles. The calculations show excellent agreement with previous experimental and numerical results, while requiring relatively short computation times. Flow predictions are also made for impinging jet configurations for which no previous data or calculations exist. (C) 2000 American Institute of Physics. [S1070-6631(00)01708-6]
\end{abstract}

\section{INTRODUCTION}

Impinging jets have most commonly been exploited for the enhanced heat and mass transfer that occurs at the impingement surface; however, the shear induced along the surface provides the impetus for several important technologies including surface cleaning ${ }^{1}$ and jet stripping ${ }^{2,3}$ in which gas jets are used to control liquid coating thickness. The induced shear can also be utilized for characterization of adhesive strength in powder coatings $s^{4}$ or trace particulate residues ${ }^{5}$ for subsequent surface sampling and chemical analysis. The ability to map the shear stress distribution for various impinging jet configurations is essential for quantification of adhesion strengths and for optimization of surface sampling or cleaning efficiency. Accurate shear stress measurements under submerged impinging jets using the electrochemical method have produced excellent agreement with a laminar boundary layer analysis close to the stagnation point. ${ }^{6-8}$ The removal of monosized microspheres from a surface during exposure to an impinging gas jet has been shown to scale with the induced wall shear stress ${ }^{9}$ and has also exhibited similar agreement with laminar boundary layer theory. ${ }^{10}$ The boundary conditions needed to determine the wall shear stress as well as heat and mass transfer to the surface are obtained from the inviscid flow just outside the boundary layer.

Although the field of jet impingement is extremely rich in the literature (see, for example, Martin ${ }^{11}$ or Looney and

\footnotetext{
a) Author to whom correspondence should be addressed. Present address: Mechanical Engineering Department, University of Delaware, Newark, DE 19716. Telephone: (302) 831-6622; Fax: (302) 831-3619. Electronic mail: phares@me.udel.edu
}

Walsh ${ }^{12}$ ), only a small number of investigators ${ }^{13-17}$ have developed inviscid flow models, thereby enabling separate treatment of the boundary layer. Strand ${ }^{13}$ obtained the flow field for potential jet impingement analytically. This solution is relevant to the impingement of a jet very close to the surface, so that impingement occurs before the jet fluid has mixed with the quiescent fluid. The inviscid impingement of fully developed laminar pipe flow was considered by Scholtz and Trass, ${ }^{14}$ who derived an analytical solution assuming a parabolic influx velocity profile. Again, this solution is valid only for small pipe to plate spacings. Parameswaran ${ }^{15}$ used a Green's function method to solve the two-dimensional stream-vorticity equation for impingement of a fully developed turbulent plane jet. Rubel ${ }^{16,17}$ developed a model of jet impingement that included turbulent mixing of the jet with the surrounding quiescent fluid by using both the fully developed and developing free jet velocity profiles for the flow into the impingement region. Rubel cast the two-dimensional and axisymmetric stream-vorticity equations into finitedifference form and solved using relaxation techniques. Although Rubel considered a wider variety of influx velocity profiles than the previous inviscid models, divergence of the iterative procedure limited the profiles to ones that included small velocity defects, while computing time limited the spatial resolution of the final solution.

An infinite number of jet velocity profiles are possible depending on the nozzle shape, height above the surface, jet Reynolds number, and Mach number. Since only a limited subset of jet velocity profiles were considered in the previous investigations, efforts to explore the effect of varying the velocity profile on technologies such as surface cleaning or sampling may be difficult. To facilitate such a study, we 


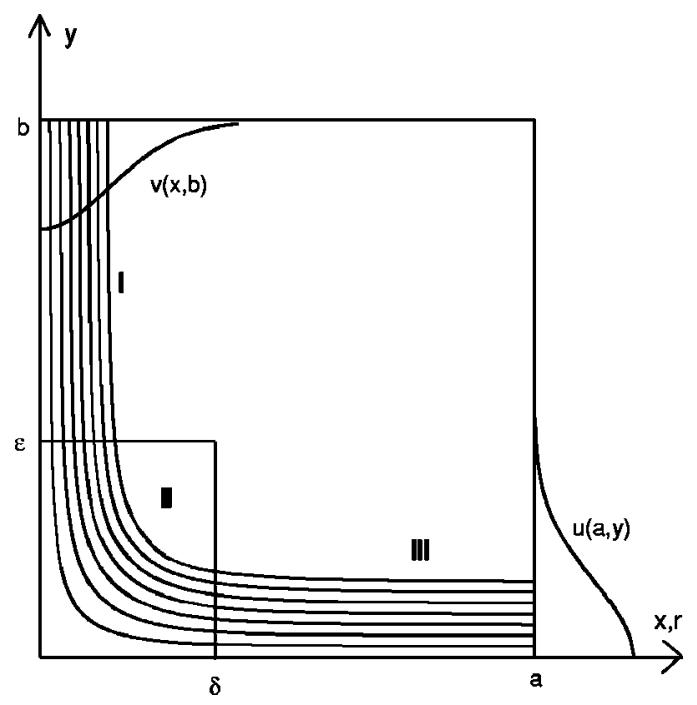

FIG. 1. Approximate view of the flow region for determining vorticity surface integral.

present a method to calculate the flowfield produced by the inviscid impingement of a two-dimensional or axisymmetric jet with an arbitrary velocity profile.

In the present work, Rubel's formulation of the problem for two-dimensional and axisymmetric inviscid jet impingement is used as a starting point. A converging infinite series solution to the governing equations is derived for an arbitrary influx stream function in terms of a surface integral of the vorticity function over the whole region of interest. Assumptions concerning the vorticity throughout the flow allow an approximate determination of this integral and, thus, a very reasonable guess of the full flowfield. By interpolating a new vorticity function distribution from the obtained stream function, the surface integral can be recalculated and a corrected solution obtained. Iteration of this process coupled with underrelaxation techniques yields quickly converging solutions. The final solutions are compared with Rubel's numerical solutions and experimental measurements of the surface pressure distributions created by fully developed and developing jet impingement. The present method of calculation requires significantly less computation time than Rubel's iterative method with modest computing power (a $233 \mathrm{MHz}$ Macintosh Powerbook G3 was employed for all of the presented calculations), while yielding stream function distributions with high spatial resolution.

Since the present method can handle influx velocity profiles with arbitrarily large velocity defects, the impingement of an annular jet profile, which resembles a fully developed round jet profile with a severe core velocity deficiency, is considered in Sec. V. The flowfield produced by the impingement of an annular jet is relevant to the interaction of V/STOL aircraft exhaust with the ground. ${ }^{18}$

\section{FORMULATION}

In accordance with Rubel's formulation all lengths and velocities are nondimensionalized with the influx velocity halfwidth and the maximum influx velocity, respectively. The coordinate system is shown in Fig. 1 with $y$ upward and perpendicular to the surface, $x$ horizontally outward for the two-dimensional case, and $r$ radially outward for the axisymmetric case. The velocity components are $u$, parallel to the surface, and $v$, perpendicular to the surface. The governing equations are

$$
\frac{\partial^{2} \psi}{\partial y^{2}}+\frac{\partial^{2} \psi}{\partial x^{2}}=\Omega(x, y)
$$

and

$$
\frac{\partial^{2} \psi}{\partial y^{2}}+\frac{\partial^{2} \psi}{\partial r^{2}}-\frac{1}{r} \frac{\partial \psi}{\partial r}=r^{2} \Omega(r, y),
$$

for two-dimensional and axisymmetric impingement, respectively. In Eq. (1), the stream and vorticity functions are defined

$$
\begin{aligned}
& \frac{\partial \psi}{\partial x}=-v, \quad \frac{\partial \psi}{\partial y}=u, \\
& \Omega=\frac{\partial u}{\partial y}-\frac{\partial v}{\partial x},
\end{aligned}
$$

and in Eq. (2)

$$
\begin{aligned}
& \frac{\partial \psi}{\partial r}=-r v, \quad \frac{\partial \psi}{\partial y}=r u, \\
& \Omega=\frac{1}{r}\left(\frac{\partial u}{\partial y}-\frac{\partial v}{\partial r}\right) .
\end{aligned}
$$

The stream function is defined to be zero along the jet centerline and the surface. The influx stream function, $F(x)$, can be determined from the given influx velocity profile at some distance, $b$, above the surface. The outflux streamlines are assumed to become parallel at some sufficiently large distance, $a$, from the jet centerline. These boundary conditions are written

$$
\begin{aligned}
& \psi(x, 0)=0, \\
& \psi(0, y)=0, \\
& \frac{\partial \psi}{\partial x}(a, y)=0, \\
& \psi(x, b)=F(x),
\end{aligned}
$$

where $x$ is simply replaced by the radial coordinate, $r$, for the axisymmetric case.

\section{ANALYTICAL SOLUTIONS}

\section{A. Two-dimensional solution}

To solve Eq. (1) analytically, given the inhomogeneous mixed boundary conditions, Eqs. (3)-(6), the general solution was assumed to be the sum of the solution, $\psi_{H}$, to the Laplace equation subject to the given boundary conditions and the solution, $\psi_{I}$, to the given Poisson equation subject to homogeneous boundary conditions. The solution to the former is found by separation of variables and application of the first three boundary conditions, Eqs. (3)-(5), yielding only one nontrivial solution, 


$$
\psi_{H}(x, y)=\sum_{n=1}^{\infty} A_{n} \sin \left(\gamma_{n} x\right) \sinh \left(\gamma_{n} y\right)
$$

where $\gamma_{n}=[(2 n-1) / 2 a] \pi$. The constants $A_{n}$ are determined by applying the last boundary condition, Eq. (6),

$$
\sum_{n=1}^{\infty} A_{n} \sin \left(\gamma_{n} x\right) \sinh \left(\gamma_{n} b\right)=F(x)
$$

Multiplying both sides of Eq. (8) by $\sin \left(\gamma_{m} x\right) d x$ and integrating from 0 to $a$ yields

$$
A_{n}=\frac{2}{a \sinh \left(\gamma_{n} b\right)} \int_{0}^{a} F\left(x^{\prime}\right) \sin \left(\gamma_{n} x^{\prime}\right) d x^{\prime} .
$$

The solution to the Poisson equation subject to homogenous mixed boundary conditions is found by expanding both the stream and vorticity functions in the following Fourier series:

$$
\begin{aligned}
& \psi_{I}(x, y)=\sum_{m=1}^{\infty} \sum_{n=1}^{\infty} C_{m n} \sin \left(\alpha_{m} y\right) \sin \left(\gamma_{n} x\right), \\
& \Omega(x, y)=\sum_{m=1}^{\infty} \sum_{n=1}^{\infty} B_{m n} \sin \left(\alpha_{m} y\right) \sin \left(\gamma_{n} x\right),
\end{aligned}
$$

where $\alpha_{m}=m \pi / b$. The Fourier coeficients, $B_{m n}$, in Eq. (11) are written as a surface integral involving the vorticity function

$$
B_{m n}=\frac{4}{a b} \int_{0}^{a} \int_{0}^{b} \Omega\left(x^{\prime}, y^{\prime}\right) \sin \left(\alpha_{m} y^{\prime}\right) \sin \left(\gamma_{n} x^{\prime}\right) d y^{\prime} d x^{\prime} \text {. }
$$

Using Eqs. (1), (10), and (11), we can solve for $C_{m n}$,

$$
C_{m n}=\frac{-B_{m n}}{\gamma_{n}^{2}+\alpha_{m}^{2}}
$$

The general solution to Eq. (1) is the sum of $\psi_{H}$ and $\psi_{I}$,

$$
\begin{aligned}
\psi(x, y)= & \sum_{n=1}^{\infty}\left[A_{n} \sinh \left(\gamma_{n} y\right)\right. \\
& \left.-\sum_{m=1}^{\infty} \frac{B_{m n}}{\gamma_{n}^{2}+\alpha_{m}^{2}} \sin \left(\alpha_{m} y\right)\right] \sin \left(\gamma_{n} x\right) .
\end{aligned}
$$

This result is the stream function distribution assuming an influx stream function profile and parallel outflux flow far from the origin. Note that the simple inviscid corner flow solution, $\psi \sim x y$, is recovered as $x$ and $y$ become very small.

Applying Eq. (14) to impinging jets requires knowledge of the vorticity function throughout the whole region so that the coefficients, $B_{m n}$, can be determined from Eq. (12). An initial guess of the vorticity function is detailed in Sec. IV A allowing calculation of the coefficients, $B_{m n}$, leading to a corrected solution.

\section{B. Axisymmetric solution}

The procedure to calculate the general solution of the axisymmetric case is identical to that presented in Sec. III A for the two-dimensional case. The resulting homogeneous stream function is

$$
\psi_{H}(r, y)=\sum_{n=0}^{\infty} D_{n} r J_{1}\left(\lambda_{n} r\right) \sinh \left(\lambda_{n} y\right)
$$

where $\lambda_{n}$ are defined such that $J_{0}\left(\lambda_{n} a\right)=0$. Again, the coefficients, $D_{n}$, are determined from the fourth boundary condition, Eq. (6),

$$
D_{n}=\frac{2}{a^{2} J_{1}^{2}\left(\lambda_{n} a\right) \sinh \left(\lambda_{n} b\right)} \int_{0}^{a} F\left(r^{\prime}\right) J_{1}\left(\lambda_{n} r^{\prime}\right) d r^{\prime} .
$$

The inhomogeneous solution, $\psi_{I}$, is determined by expanding the stream function and the right-hand side of Eq. (2) into the following series:

$$
\begin{aligned}
& \psi_{I}(r, y)=\sum_{m=1}^{\infty} \sum_{n=1}^{\infty} F_{m n} r J_{1}\left(\lambda_{n} r\right) \sin \left(\alpha_{m} y\right), \\
& r^{2} \Omega(r, y)=\sum_{m=1}^{\infty} \sum_{n=1}^{\infty} E_{m n} r J_{1}\left(\lambda_{n} r\right) \sin \left(\alpha_{m} y\right),
\end{aligned}
$$

where, once again, a surface integral involving the vorticity function is embedded in the series coefficients, $E_{m n}$,

$$
\begin{aligned}
E_{m n}= & \frac{4}{b a^{2} J_{1}^{2}\left(\lambda_{n} a\right)} \int_{0}^{a} \int_{0}^{b} r^{\prime} 2 \Omega\left(r^{\prime}, y^{\prime}\right) J_{1}\left(\lambda_{n} r^{\prime}\right) \\
& \times \sin \left(\alpha_{m} y^{\prime}\right) d y^{\prime} d r^{\prime} .
\end{aligned}
$$

Using Eqs. (2), (17), and (18),

$$
F_{m n}=\frac{-E_{m n}}{\alpha_{m}^{2}+\lambda_{n}^{2}}
$$

The general solution to Eq. (2) subject to the boundary conditions, Eqs. (3)-(6), is

$\psi(r, y)$

$$
=\sum_{n=0}^{\infty}\left[D_{n} \sinh \left(\lambda_{n} y\right)-\sum_{m=0}^{\infty} \frac{E_{m n}}{\alpha_{m}^{2}+\lambda_{n}^{2}} \sin \left(\alpha_{m} y\right)\right] r J_{1}\left(\lambda_{n} r\right) .
$$

\section{METHOD OF CALCULATION}

\section{A. Initial guess}

To apply Eqs. (14) and (21) to the physical problem of impingement flow, the surface integrals in Eqs. (12) and (19) must be evaluated. Therefore, some information about how the vorticity function behaves within the region of interest is required. Due to the inviscid approximation, the vorticity is constant along streamlines; so $\Omega=\Omega(\psi)$. This alone is not sufficient to solve the surface integral; however, if we also assume that the influx velocity halfwidth is small compared to the integration limits, $a$ and $b$, then we can approximate the stream function (and thus the vorticity function) over most of the region with a far-field expression. 
The small near-field region close to the intersection of the jet centerline and the surface must be treated separately. Hence an initial guess is formulated by splitting the surface integrals into the three regions portrayed in Fig. 1: the inflow region, I, and the outflow region, III, which require far-field solutions, and the impingement region, II, which requires a near-field solution.

\section{Far-field solutions}

The approximation for region I stems from the observation that the influx near the centerline does not sense the presence of the surface until it is very close to the surface. ${ }^{19}$ The vorticity function is, therefore, determined by the influx condition and can be expressed in terms of the influx stream function, $F(x)$, as

$$
\begin{aligned}
& \Omega_{\mathbf{I}}(x)=F^{\prime \prime}(x), \\
& \Omega_{\mathbf{I}}(r)=\frac{F^{\prime \prime}(r)}{r^{2}}-\frac{F^{\prime}(r)}{r^{3}},
\end{aligned}
$$

for the two-dimensional and axisymmetric cases, respectively.

Similar to the reasoning for region $\mathbf{I}$, we assume that the vorticity close to the surface in region III approaches the outflux condition very soon after redirection. This condition is a little harder to implement directly since the outflux boundary condition only assumes parallel streamlines. Therefore, we refer to Rubel's method ${ }^{16}$ for converting the mixed boundary conditions to Dirichlet boundary conditions. For the two-dimensional case, Rubel showed that Eqs. (1) and (5) imply that the influx stream function profile is identical to the outflux stream function profile. Thus $\psi(a, y)$ $=F(y)$ and the vorticity function in region III is taken to be

$$
\Omega_{\text {III }}(y)=F^{\prime \prime}(y),
$$

for the two-dimensional case.

Similarly, Rubel showed that Eqs. (2) and (5) imply that the influx and outflux stream function are parametrically related such that $\psi(a, y)=F(\sqrt{2 a y})$. Since the outflux stream function profile depends on the radial location of the boundary, $a$, it seems reasonable that, as the outflux condition is approached for large radial distances, the far-field stream function could be written as $\psi(r, y)=F(\sqrt{2 r y})$, which approaches the parallel outflux condition far from the origin. Therefore, we have for the axisymmetric case

$$
\Omega_{\text {III }}(r, y)=\frac{F^{\prime \prime}(\xi)}{\xi^{2}}-\frac{F^{\prime}(\xi)}{\xi^{3}},
$$

where $\xi=\sqrt{2 r y}$.

\section{Near-field solutions}

The vorticity function in region II is determined from the stream function behavior close to the origin. As mentioned in Sec. III, $\psi \sim x y$ for small $x$ and $y$ in the twodimensional case. Since vorticity is constant along streamlines, then $\Omega$ is a function of $x y$,

$$
\Omega(x, y)=\Omega[G(x y)],
$$

where the function $G$ is determined by matching the vorticity function at $x=\delta$ and $y=\epsilon$ with the far-field solutions (see Fig. 1). Since $\Omega(x, \delta)=F^{\prime \prime}(x)$ and $\Omega(\epsilon, y)=F^{\prime \prime}(y)$, then

$$
G(x \delta)=x, \quad G(\epsilon y)=y,
$$

and thus

$$
\Omega_{\mathbf{I I}}(x, y)=F^{\prime \prime}\left(\frac{x y}{\epsilon}\right),
$$

for $\delta=\epsilon$.

For the axisymmetric case, $\psi \sim r^{2} y$ for small $r$ and $y$; and matching the vorticity function at $\delta$ and $\epsilon$ yields

$$
\Omega_{\mathbf{I I}}(r, y)=\frac{F^{\prime \prime}(\zeta)}{\zeta^{2}}-\frac{F^{\prime}(\zeta)}{\zeta^{3}},
$$

for $\delta=2 \epsilon$, where $\zeta=r \sqrt{\frac{y}{\epsilon}}$.

\section{The total integral}

Since we have estimated the value of the vorticity function in the three regions, the surface integral in Eqs. (12) and (19) can be split into three integrals of known functions. For the two-dimensional case, we have

$$
\begin{aligned}
& \int_{0}^{a} \int_{0}^{b} \Omega\left(x^{\prime}, y^{\prime}\right) \sin \left(\alpha_{m} y^{\prime}\right) \sin \left(\gamma_{n} x^{\prime}\right) d y^{\prime} d x^{\prime} \\
& \quad=I_{1}+I_{2}+I_{3},
\end{aligned}
$$

where

$$
\begin{aligned}
& I_{1}=\int_{0}^{a} \int_{\epsilon}^{b} F^{\prime \prime}\left(x^{\prime}\right) \sin \left(\alpha_{m} y^{\prime}\right) \sin \left(\gamma_{n} x^{\prime}\right) d y^{\prime} d x^{\prime}, \\
& I_{2}=\int_{0}^{\epsilon} \int_{0}^{\epsilon} F^{\prime \prime}\left(\frac{x^{\prime} y^{\prime}}{\epsilon}\right) \sin \left(\alpha_{m} y^{\prime}\right) \sin \left(\gamma_{n} x^{\prime}\right) d y^{\prime} d x^{\prime}, \\
& I_{3}=\int_{\epsilon}^{a} \int_{0}^{b} F^{\prime \prime}\left(y^{\prime}\right) \sin \left(\alpha_{m} y^{\prime}\right) \sin \left(\gamma_{n} x^{\prime}\right) d y^{\prime} d x^{\prime} .
\end{aligned}
$$

These integrals can be computed numerically for an arbitrary influx stream function, $F(x)$. Fortunately, only $I_{2}$ requires a two-dimensional integration, since $I_{1}$ can be integrated directly with respect to $y$; and $I_{3}$, with respect to $x$. Note that the $I_{1}$ and $I_{3}$ integrals overlap where $x, y>\epsilon$, but at least one of the vorticity functions is essentially zero in this region.

The computation of the surface integral in the axisymmetric case is more time consuming than the twodimensional case, since the corresponding $I_{3}$ integral requires integration over both $r$ and $y$

$$
\begin{aligned}
\int_{0}^{a} \int_{0}^{b} r^{\prime 2} \Omega\left(r^{\prime}, y^{\prime}\right) J_{1}\left(\delta_{n} r^{\prime}\right) \sin \left(\alpha_{m} y^{\prime}\right) d y^{\prime} d r^{\prime} \\
=I_{1}+I_{2}+I_{3} \\
I_{1}=\int_{0}^{a} \int_{\epsilon}^{b}\left(F^{\prime \prime}\left(r^{\prime}\right)-\frac{F^{\prime}\left(r^{\prime}\right)}{r^{\prime}}\right) \\
\quad \times J_{1}\left(\lambda_{n} r^{\prime}\right) \sin \left(\alpha_{m} y^{\prime}\right) d y^{\prime} d r^{\prime}
\end{aligned}
$$




$$
\begin{aligned}
I_{2}= & \int_{0}^{\delta} \int_{0}^{\epsilon}\left(F^{\prime \prime}\left(r^{\prime} \sqrt{\frac{y^{\prime}}{\epsilon}}\right)-\frac{F^{\prime}\left(r^{\prime} \sqrt{\frac{y^{\prime}}{\epsilon}}\right)}{r^{\prime} \sqrt{\frac{y^{\prime}}{\epsilon}}}\right) \\
& \times J_{1}\left(\lambda_{n} r^{\prime}\right) \sin \left(\alpha_{m} y^{\prime}\right) d y^{\prime} d r^{\prime}, \\
I_{3}= & \int_{\delta}^{a} \int_{0}^{b}\left(F^{\prime \prime}\left(\sqrt{2 r^{\prime} y^{\prime}}\right)-\frac{F^{\prime}\left(\sqrt{2 r^{\prime} y^{\prime}}\right)}{\sqrt{2 r^{\prime} y^{\prime}}}\right) \\
& \times J_{1}\left(\lambda_{n} r^{\prime}\right) \sin \left(\alpha_{m} y^{\prime}\right) d y^{\prime} d r^{\prime} .
\end{aligned}
$$

For all cases considered in the present study, the terms of the summations in Eqs. (14) and (21) were calculated until they fell below $0.01 \%$ of the maximum stream function value. This required calculation of roughly 15 terms of the outer summation, and a maximum of 50 terms of the inner summation. The number of terms calculated in the inner summation decreased with each subsequent term of the outer summation (i.e., only one term of the inner summation needed to be calculated for the last term calculated for the outer summation).

\section{B. Iteration and convergence}

The far-field/near-field stream function approximations detailed in the previous sections combined with Eqs. (14) and (21), for the two-dimensional and the axisymmetric cases, respectively, provide an initial guess of the flow field. The obtained stream function distribution can be converted to a vorticity function distribution using the known correlation between the vorticity and stream function at the influx boundary, allowing the vorticity integral to be reevaluated and a new corrected solution obtained. Repeated correction of the stream function distribution by this method yields a converging solution under certain conditions. For example, it was found that the iteration must be performed with strong underrelaxation, i.e.,

$$
\psi^{\nu+1}=(1-\theta) \psi^{\nu}+\theta \psi^{*},
$$

where $\theta \leqslant 0.3, \nu$ is the iteration number, and $\psi^{*}$ is the intermediate stream function obtained from direct correction of $\psi^{\nu}$. Figure 2 shows the effect of the relaxation parameter, $\theta$, on the residual, $R$, of the iteration for the two-dimensional case, where

$$
R=\left|\psi^{\nu+1}-\psi^{\nu}\right|_{\max } .
$$

Figure 3 demonstrates that the initial guess presented in Sec. IV A is important, since convergence is achieved only for certain matching locations, $\epsilon$. A value of $\epsilon=1.1$ produces a reasonable initial guess for two-dimensional impingement that converges rapidly to the final solution. A similar analysis of axisymmetric impingement suggests that $\epsilon=0.5$ is a suitable matching location. Other than affecting the convergence rate, $\epsilon$ has no effect on the final solution.

In the present study, iteration by this method was continued until $R \leqslant 10^{-3}$. At this prescribed tolerance, another order of magnitude reduction in the residual produced a less than $10^{-3}$ maximum variation in the velocity at the ground

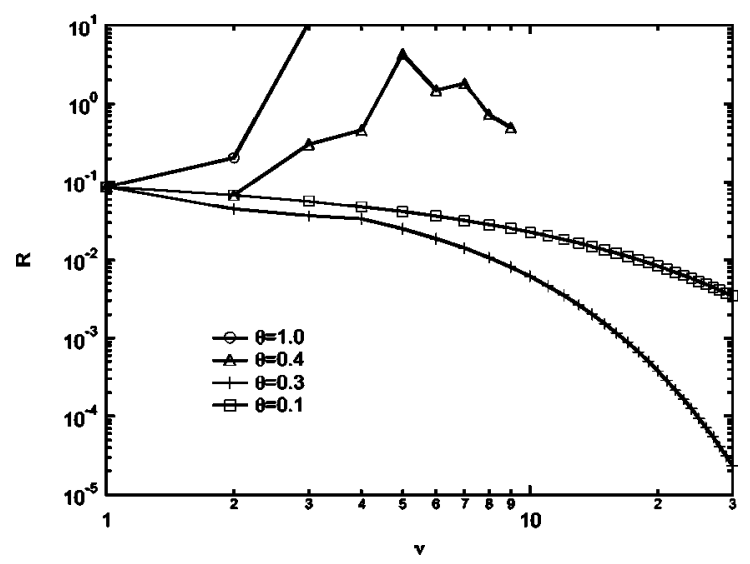

FIG. 2. Effect of relaxation on solution convergence.

plane $(y=0)$ and the outflux stream function profile deviated from Rubel's Dirichlet conditions by less than $1 \%$.

\section{RESULTS AND DISCUSSION}

The discussion that follows compares published surface pressure data with the present calculations. The surface pressure is determined from Bernoulli's equation, i.e.,

$$
P(x)=1-\left(\left.\frac{\partial \psi}{\partial y}\right|_{y=0}\right)^{2}
$$

and

$$
P(r)=1-\left(\left.\frac{1 \partial \psi}{r \partial y}\right|_{y=0}\right)^{2},
$$

for the two-dimensional and axisymmetric cases, respectively.

\section{A. Two-dimensional jet}

\section{Fully developed jet impingement}

Surface pressure measurements for the impingement of fully developed two-dimensional jets were made by Schauer and Eusits, ${ }^{20}$ Kumada and Mabuchi, ${ }^{21}$ and Beltaos and Rajaratnam $^{22}$ for a variety of jet heights, $H$, and jet Reynolds numbers. A comparison between their experimental data and

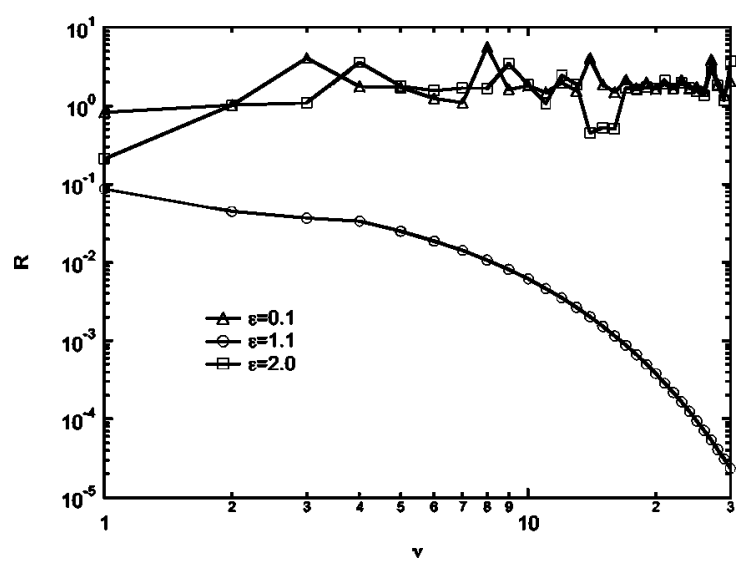

FIG. 3. Effect of initial guess on solution convergence. 


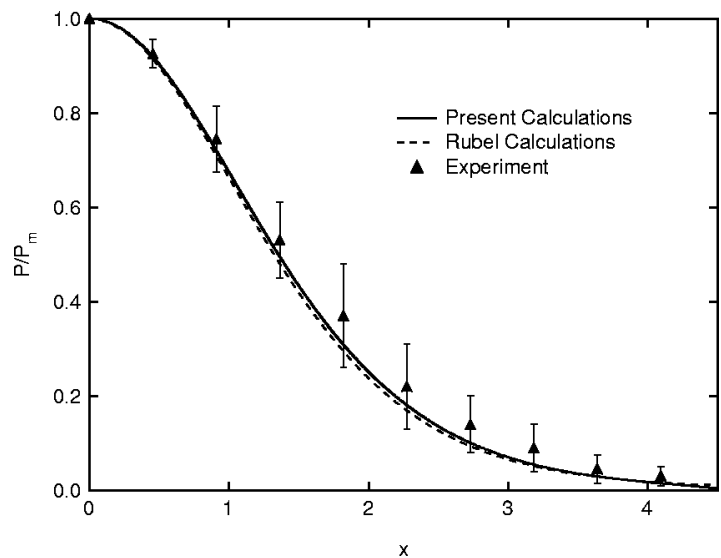

FIG. 4. Comparison of predicted surface pressure distribution with experimental and numerical results for an impinging two-dimensional jet.

the calculated surface pressure distribution, normalized with the maximum stagnation pressure, $P_{m}$, is shown in Fig. 4. The experimental data collapse to a single curve when distances are scaled with $H$. In order to convert to the present convention, it was necessary to determine the velocity halfwidth, $b_{u}$, at the prescribed influx location. For a free jet, Beltaos and Rajaratnam ${ }^{22}$ found

$$
\frac{b_{u}}{H}=0.10\left(\frac{y^{\star}}{H}+0.15\right) \text {, }
$$

where $y^{\star}$ is the dimensional distance from the jet nozzle. Furthermore, it was found that the influx location-taken to be the surface of departure of the jet from free jet behavior -is consistently located at $0.7 \mathrm{H}$ from the nozzle. Therefore, conversion simply involved dividing distances by the factor $b_{u} / H=0.085$, and the collapse remains.

The solid line in Fig. 4 represents the predicted pressure distribution. The values of the integration limits, $a$ and $b$, were chosen such that any increase in the these values had no effect on the solution. As observed by Rubel, the values $a$ $=5$ and $b=5$ placed the boundaries sufficiently far from the origin. Choosing the boundaries in this manner suggests unconfined jet impingement- the absence of a top surface that would constrict the flow and thus affect the surface pressure distribution. The fully developed two-dimensional free jet velocity profile derived by Gortler ${ }^{23}$ was employed for the influx profile, i.e.,

$$
-v(x)=\left(1-\tanh ^{2}(c x)\right),
$$

where $c=\tanh ^{-1}(1 / \sqrt{2})$.

The pressure distribution shown as a dashed line in Fig. 4 was obtained using the numerical method of Rubel. ${ }^{16}$ In accordance with his suggestions, a uniform $41 \times 41$ grid spanning a $5 \times 5$ square was employed for the finite difference calculations. The maximum deviation between the two methods of calculation is $1 \%$ and occurs at a location of $x$ $=1.6$.

Figure 5 compares the predicted streamlines for an impinging, fully developed two-dimensional jet using both methods of calculation. The parallel outflux boundary condition, which could only be approximated by an outflux stream

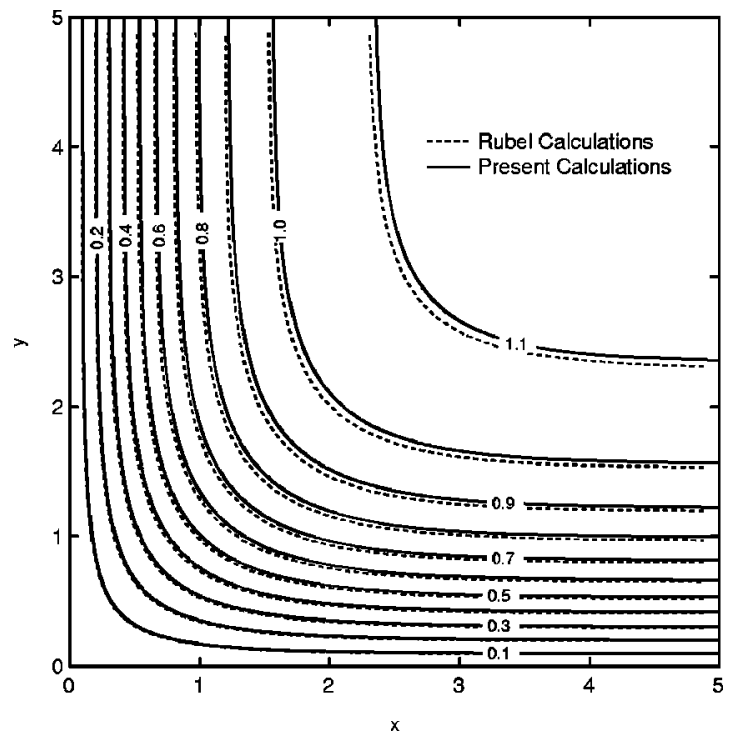

FIG. 5. Two-dimensional impinging jet streamlines as calculated using Rubel method and the present method.

function distribution in Rubel's calculations, is met exactly in the present calculations. This is evident in Fig. 5, especially for the higher valued streamlines.

\section{Developing jet impingement}

When a turbulent jet is located less than about 6 jet widths above the surface $(H / D<6)$, then the jet potential core will impinge upon the surface. Since the jet velocity profile is no longer self-similar due to the uniform velocity of the potential core, it would not be surprising if the form of the surface pressure profile deviated from similarity. In contrast, $\mathrm{Tu}$ and $\mathrm{Wood}^{2}$ observed pressure profile similarity for unconfined jet heights as low as $H / D=1$, when lengths were scaled with $D$. Converting the data to the present convention involved scaling distances with $b_{u}$ which slightly increases with downstream distance. ${ }^{10}$ Thus, unlike the fully developed case, similarity breaks down in the present convention for developing jet impingement.

The experiments of $\mathrm{Tu}$ and $\mathrm{Wood}^{2}$ include conditions that allow direct comparison with Rubel's ${ }^{16}$ numerical re-

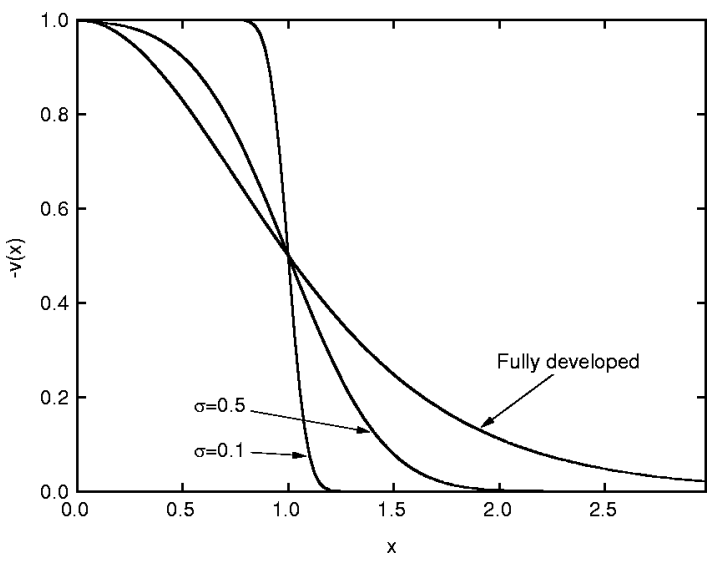

FIG. 6. Influx velocity profiles used for calculations. 


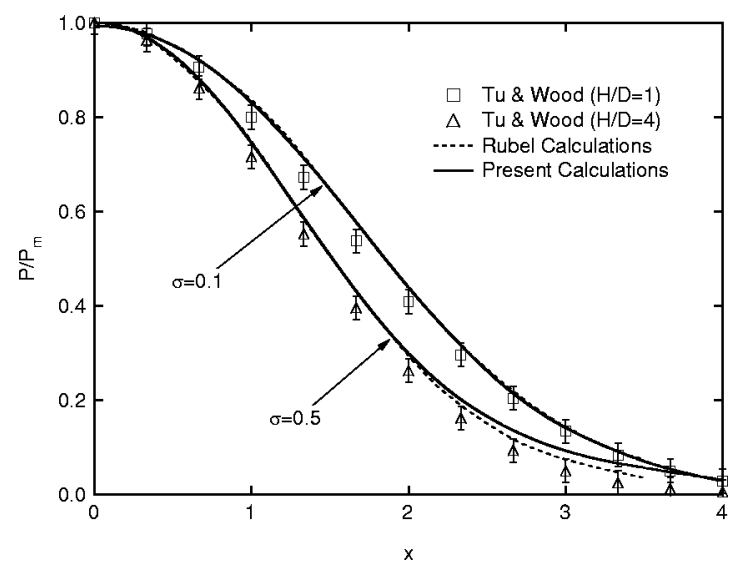

FIG. 7. Observed and predicted surface pressure distributions for twodimensional developing jet impingement for $\sigma=0.1$ and $\sigma=0.5$.

sults. To facilitate comparison of the present model with that of Rubel, we employ the developing jet velocity profile used in the previous calculations,

$$
-v(x)=\frac{1}{2}\left[1+\operatorname{erf}\left(\frac{1-x}{\sigma}\right)\right]
$$

where $\sigma$ is a spreading parameter. The difference between this velocity profile and that for the fully developed jet is shown in Fig. 6. Figure 7 compares the surface pressure distributions predicted by the two models with the experimentally obtained pressure profiles of $\mathrm{Tu}$ and $\mathrm{Wood}^{2}$ for $H / D$ $=1$ and $H / D=4$. From the empirical observations by Reichardt $^{23}$ of the mixing zone in a developing twodimensional free jet, these jet heights would roughly correspond to influx velocity profiles with spreading parameters of $\sigma=0.1$ and $\sigma=0.5$, respectively. The predicted widening of the pressure profile due to potential core impingement is in good agreement with the presented data.

\section{B. Axisymmetric jet}

A major difference between treatment of the twodimensional jet and the axisymmetric jet is the handling of the parallel outflux boundary condition. It was shown in Sec. IV A 1 that parallel outflux implies an essentially parallel farfield flow for the two-dimensional case, whereas the far-field streamlines resemble hyperbolas for the axisymmetric case. The hyperbolic streamlines approach the parallel outflux condition infinitely far from the origin, so placement of the outflux boundary at a finite radial location results in a premature parallelization of the outflux streamlines. This, in turn, decreases the velocity approaching the boundary. To ensure that this effect does not impact the region of strong pressure gradient $(r<4)$, the outflux boundary must be farther from the origin than in the two-dimensional case. Systematic variation of the boundary locations revealed that a radial location of $a=10$ was sufficient for the present calculations.

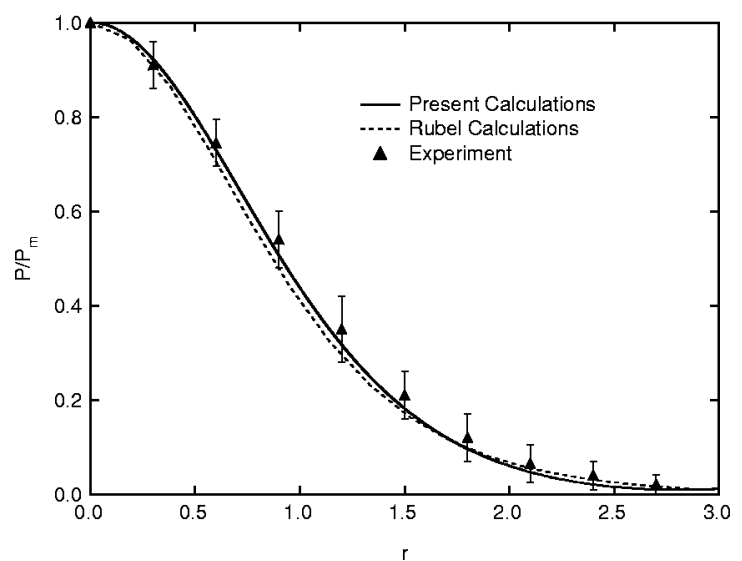

FIG. 8. Comparison of predicted surface pressure distribution with experimental and numerical results for a fully-developed impinging axisymmetric jet.

\section{Fully developed jet impingement}

The Schlichting similarity solution ${ }^{23}$ for a fully developed, axisymmetric free jet is used to define the influx velocity profile, i.e.,

$$
-v(r)=\frac{1}{\left(1+(\sqrt{2}-1) r^{2}\right)^{2}} .
$$

This similarity solution is applicable to jet impingement for $H / D>8 .{ }^{24}$ Surface pressure measurements for normally impinging axisymmetric jets at these heights were made by Bradbury $^{25}(12<H / D<20)$, Beltaos and Rajaratnam ${ }^{19}$ (20 $<H / D<66)$, and Giralt, Chia, and $\operatorname{Trass}^{24}(8<H / D<20)$. These data are presented in Fig. 8 along with the calculated surface pressures using the present method and Rubel's numerical method. All are in excellent agreement.

\section{Developing jet impingement}

Similarity of the surface pressure profiles breaks down when the axisymmetric jet potential core impinges onto the surface $(H / D<8) .{ }^{24}$ Furthermore, we expect that the pressure profile should approach that predicted by the inviscid

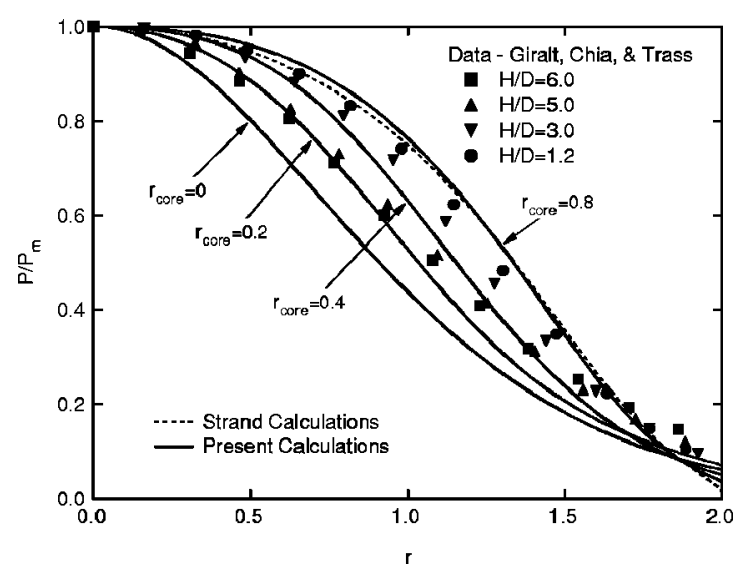

FIG. 9. Measurements of Giralt, Chia, and Trass (1977) for various jet heights compared with present calculations for developing jet impingement and Strand's (1964) potential jet solution. 


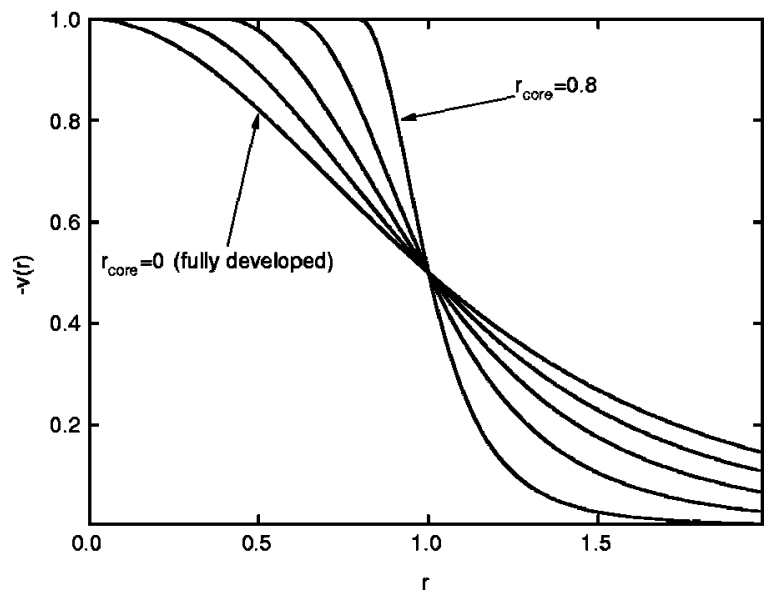

FIG. 10. Developing jet velocity profiles.

impinging potential jet model of Strand ${ }^{13}$ as the jet height is decreased. Surface pressure measurements for impinging developing jets have been made by Giralt, Chia, and Trass ${ }^{24}$ for several jet heights in the range $1.2<H / D<6.0$. These data are presented in Fig. 9 along with Strand's solution and the present calculations for several values of the potential core radius, $r_{\text {core }}$. Note the good agreement between the data at the lowest jet height, Strand's potential solution, and the calculations for the widest core radius. The developing jet velocity profiles used for the calculations were defined such that $u=1$ for $r \leqslant r_{\text {core }}$ and with Eq. (38) describing the shape of the mixing zone $\left(r>r_{\text {core }}\right)$. These profiles are presented in Fig. 10.

\section{Stagnation bubbles and annular jet impingement}

The shock waves produced within the potential core of an underexpanded free jet cause a decrease in the centerline jet velocity. ${ }^{26}$ The velocity profile downstream will resemble the fully developed profile described in Eq. (38) with a velocity deficient core. The impingement of this type of profile was also investigated numerically by Rubel, ${ }^{17}$ who predicted the conditions at which areas of recirculation would develop near the stagnation point. Rubel used a family of velocity profiles described in terms of the location of the maximum velocity, $r_{m}$, and the core velocity deficiency, $d_{w}$-the difference between the maximum and centerline velocities. These profiles were defined such that the centerline vorticity was nonzero for $d_{w}>0$, i.e.,

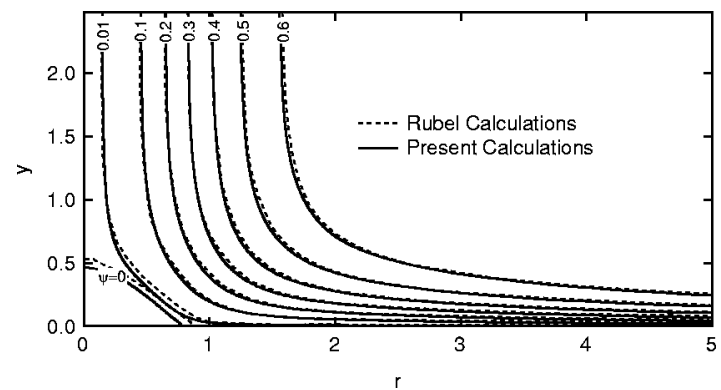

FIG. 11. Streamline plot with stagnation bubble for $r_{m}=0.35$ and $d_{w}$ $=0.16$.

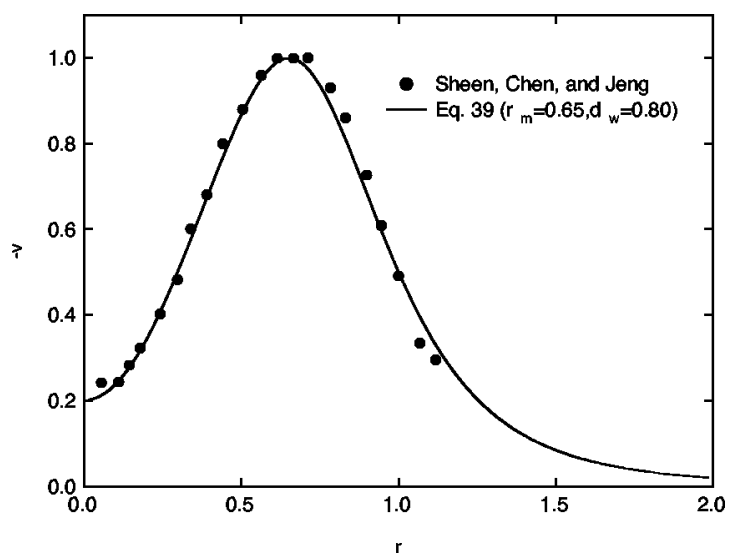

FIG. 12. Comparison of annular free jet velocity data at a downstream distance one outer diameter with Eq. (39).

$$
\begin{aligned}
& -v(r)=1-d_{w}+2 d_{w} \frac{r^{2}}{r_{m}^{2}}\left(1-\frac{1 r^{2}}{2 r_{m}^{2}}\right), \quad 0 \leqslant r \leqslant r_{m}, \\
& -v(r)=\left(1+c_{m}\left(r-r_{m}\right)^{2}\right)^{-2}, \quad r \geqslant r_{m},
\end{aligned}
$$

where $c_{m}=(\sqrt{2}-1) /\left(1-r_{m}\right)^{2}$. Using these profiles, Rubel found recirculation regions for $d_{w} / r_{m}>0.22$, but was unable to find solutions for $d_{w} / r_{m}>0.47$. The present method yields results similar to Rubel for $d_{w} / r_{m}<0.47$. Solutions are also possible for $d_{w} / r_{m}>0.47$. Figure 11 compares streamlines calculated with the present method with those calculated using Rubel's method ${ }^{17}$ for $r_{m}=0.35$ and $d_{w}=0.16$. The stagnation bubble appears as the area within the dividing streamline corresponding to $\psi=0$.

Further increase in the core deficiency produces a velocity profile that resembles the near-nozzle profile of a free annular jet. Figure 12 compares the profile calculated using Eq. (39) $\left(r_{m}=0.65, d_{w}=0.80\right)$ with the free annular jet velocity data of Sheen, Chen, and Jeng ${ }^{27}$ at a downstream distance of about one outer diameter. In this case, the inner diameter was close to one-half of the outer diameter. Because of the severe core velocity deficiency $\left(d_{w} / r_{m}\right.$ $=1.23$ ), the impingement of such a flow could not be treated with Rubel's method. Figure 13 depicts streamlines calculated with the present method for this case. Since no experimental data was found in the literature on the size of the recirculating region under an impinging annular jet, comparison with measurements is not possible. However, some pre-

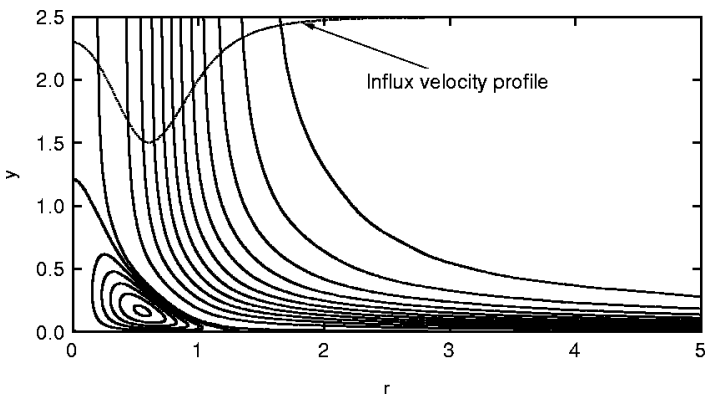

FIG. 13. Streamline plot with overlaid influx velocity profile for annular jet impingement $\left(r_{m}=0.65\right.$ and $\left.d_{w}=0.80\right)$. 


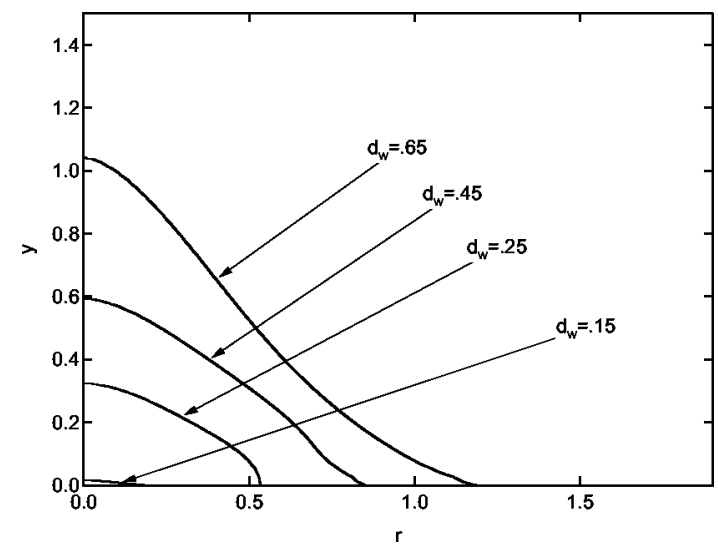

FIG. 14. Shape of recirculation zone for various core velocity deficiencies $\left(r_{m}=0.60, d_{w}=0.15,0.25,0.45,0.65\right)$.

dictions of the shape of the recirculation region are presented in Fig. 14, which depicts the streamlines corresponding to $\psi=0$ for several core velocity deficiencies. A plot of the locations of the dividing streamline along the jet centerline and the surface ( $y_{d}$ and $r_{d}$, respectively) against the relative core velocity deficiency, $d_{w} / r_{m}$, for $r_{m}=0.3$ and $r_{m}=0.6$ is presented in Fig. 15. The critical value of the relative core velocity deficiency leading to formation of a recirculation region agrees with Rubel's predicted value of $d_{w} / r_{m}$ $=0.22$. The value of $r_{d}$ could easily be determined experimentally, as it would be accompanied by a maximum in the surface pressure profile. Experimental verification of these predictions would require knowledge of the velocity profile at the top of the impingement region. Reasonable agreement with experiment would confirm the validity of inviscid models for the impingement region of annular and underexpanded jet impingement on a flat surface, and would support Rubel's ${ }^{17}$ suggestion that the inviscid total pressure defect mechanism is responsible for the observed stagnation bubbles under impinging underexpanded jets.

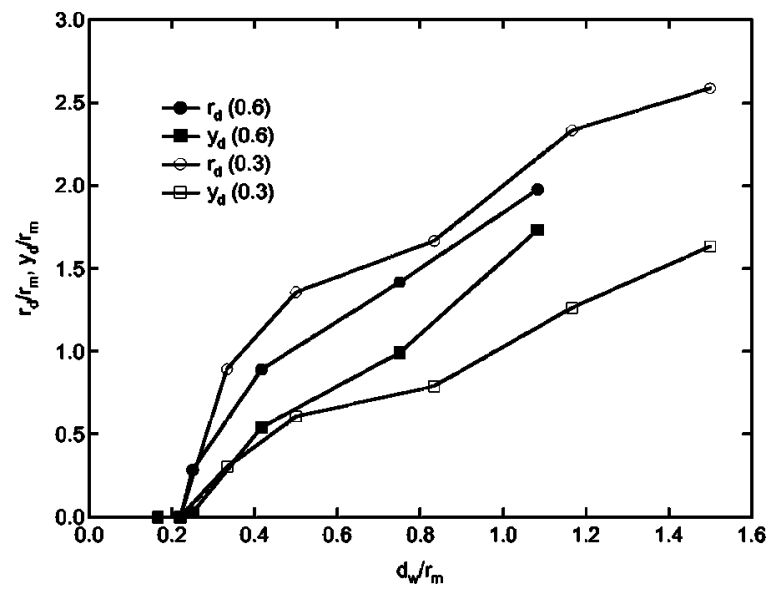

FIG. 15. Predicted dependence of dividing streamline location on core deficiency for $r_{m}=0.3,0.6$.

\section{CONCLUSION}

A method for calculating the flow field for axisymmetric and two-dimensional inviscid impingement flow is presented. Expressions for the stream function were derived in terms of the vorticity function distribution which was approximated by matching far-field and near-field expressions at prescribed locations, thus yielding a corrected solution. Successive correction of the stream function distribution resulted in a quickly converging solution. The method was applied to flow calculations for various two-dimensional and axisymmetric impinging jet configurations, including annular jet impingement, which has not been considered in previous inviscid calculations. The accuracy of the present calculations is sufficient to demonstrate agreement with previous numerical results and with the available data.

\section{ACKNOWLEDGMENT}

This work was supported by the Federal Aviation Administration under Grant No. FAA-93-G-060.

${ }^{1}$ R. Sherman, J. Grob, and W. Whitlock, "Dry surface cleaning using carbon dioxide snow," J. Vac. Sci. Technol. A 9, 1970 (1991).

${ }^{2} \mathrm{C}$. V. Tu and D. H. Wood, "Wall shear stress measurements beneath an impinging jet," Exp. Therm. Fluid Sci. 13, 364 (1996).

${ }^{3}$ M. Bouainouche, N. Bourabaa, and B. Desmet, "Numerical study of the wall shear stress produced by the impingement of a plane turbulent jet on a plate,"' Int. J. Numer. Methods Heat Fluid Flow 7, 548 (1997).

${ }^{4}$ B. E. Russ and J. B. Talbot, "A method for measuring the adhesion strength for powder coatings," J. Adhes. 68, 257 (1998).

${ }^{5}$ D. J. Phares, J. K. Holt, G. T. Smedley, and R. C. Flagan, "Method for characterization of adhesion properties of trace explosives in fingerprints and fingerprint simulations,' J. Forensic Sci. 45, 762 (2000).

${ }^{6} \mathrm{~K}$. Kataoka and T. Mizushina, "Local enhancement of the rate of heat transfer in an impinging round jet by free-stream turbulence," in Proceedings 5th International Heat Transfer Conference, page FC8.3, 1974.

${ }^{7}$ K. Kataoka, Y. Kamiyama, S. Hashimoto, and T. Komai, "Mass transfer between a plane surface and an impinging jet,"' J. Fluid Mech. 119, 91 (1982).

${ }^{8}$ S. V. Alekseenko and D. M. Markovich, "Electrodiffusion diagnostics of wall shear stresses in impinging jets," J. Appl. Electrochem. 24, 626 (1994).

${ }^{9}$ G. T. Smedley, D. J. Phares, and R. C. Flagan, "Entrainment of fine particles from surfaces by gas jets impinging at normal incidence," Exp. Fluids 26, 324 (1999).

${ }^{10}$ D. J. Phares, G. T. Smedley, and R. C. Flagan, "The wall shear stress produced by the normal impingement of a jet on a flat surface," J. Fluid Mech. (in press).

${ }^{11} \mathrm{H}$. Martin, "Heat and mass transfer between impinging gas jets and solid surfaces," Adv. Heat Transfer 13, 1 (1977).

${ }^{12}$ M. K. Looney and J. J. Walsh, "Mean-flow and turbulent characteristics of free and impinging jet flows," J. Fluid Mech. 147, 397 (1984).

${ }^{13} \mathrm{~T}$. Strand, "On the theory of normal ground impingement of axisymmetric jets in inviscid incompressible flow," Paper 64-424, AIAA, New York, 1964.

${ }^{14} \mathrm{M}$. T. Scholtz and O. Trass, "Mass transfer in a nonuniform impinging jet," AIChE J. 16, 82 (1970).

${ }^{15}$ V. Parameswaran, "Study of reattaching wall jets," Ph.D. thesis, Dept. Mech. Eng. University of Waterloo, Ontario, Canada, 1973.

${ }^{16}$ A. Rubel, "Computations of jet impingement on a flat surface,' AIAA J. 18, 168 (1980).

${ }^{17} \mathrm{~A}$. Rubel, "Inviscid axisymmetric jet impingement with recirculating stagnation regions," AIAA J. 21, 351 (1983).

${ }^{18}$ J. M. Kuhlman and W. M. Cavage, "Jet ground vortex formation by annular jets,” J. Aircr. 31, 794 (1994).

${ }^{19} \mathrm{~S}$. Beltaos and N. Rajaratnam, "Circular turbulent impinging jets," J. Hydraul. Div., Am. Soc. Civ. Eng. 100(HY10), 1313 (1974).

${ }^{20}$ J. J. Schauer and R. H. Eustis, “The flow development and heat transfer 
characteristics of plane turbulent impinging jets," Tech Report 3, Dept. Mech. Eng., Stanford University, Stanford, CA, 1963.

${ }^{21}$ M. Kumada and I. Mabuchi, "Studies on the heat transfer of impinging jet," Bull. JSME 13, 77 (1970).

${ }^{22}$ S. Beltaos and N. Rajaratnam, "Plane turbulent impinging jets," J. Hydraul. Res. 11, 29 (1973).

${ }^{23}$ H. Schlichting, Boundary Layer Theory (McGraw-Hill, New York, 1960).

${ }^{24} \mathrm{~F}$. Giralt, C. Chia, and O. Trass, "Characterization of the impingement region in an axisymmetric turbulent jet,' Ind. Eng. Chem. Fundam. 16, 21 (1977).

${ }^{25}$ L. J. S. Bradbury, "The impact of an axisymmetric jet onto a normal ground," Aeronaut. Q. 23, 141 (1972).

${ }^{26}$ C. D. Donaldson and R. S. Snedeker, "Mean properties of free and impinging jets," J. Fluid Mech. 45, 281 (1971).

${ }^{27}$ H. J. Sheen, W. J. Chen, and S. Y. Jeng, "Recirculation zones of unconfined and confined annular swirling jets," AIAA J. 34, 572 (1996). 